\title{
Size-Selective Carbon Nanoclusters as Precursors to the Growth of Epitaxial Graphene
}

\author{
Bo Wang, ${ }^{\dagger, \S}$ Xiufang Ma, ${ }^{\ddagger} \S$ Marco Caffio, ${ }^{\dagger}$ Renald Schaub, ${ }^{*}{ }^{\dagger}$ and Wei-Xue Li ${ }^{*}, \ddagger$
}

†Scottish Centre for Interdisciplinary Surface Spectroscopy, School of Chemistry, University of St Andrews, St Andrews, KY16 9ST, United Kingdom

¥State Key Laboratory of Catalysis and Center for Theoretical and Computational Chemistry, Dalian Institute of Chemical Physics, Chinese Academy of Sciences, Dalian 116023, China

Corresponding Authors $(*)$ :

Author Contributions (§): renald.schaub@st-andrews.ac.uk; wxli@dicp.ac.cn.

These authors have contributed equally.

The nucleation and growth mechanisms of graphene on $\mathrm{Rh}(111)$ via temperature-programmed growth of $\mathrm{C}_{2} \mathrm{H}_{4}$ are studied by scanning tunneling microscopy and spectroscopy, and by density functional theory calculations. By combining our experimental and first principles approaches, we show that carbon nanoislands form in the initial stages of graphene growth, possessing an exclusive size of seven honeycomb carbon units (hereafter labeled as $7 C_{6}$ ). These clusters adopt a domelike hexagonal shape indicating that bonding to the substrate is localized on the peripheral $\mathrm{C}$ atoms. Smoluchowski ripening is identified as the dominant mechanism leading to the formation of graphene, with the size-selective carbon islands as precursors. Control experiments and calculations, whereby coronene molecules, the hydrogenated analogues of $7 C_{6}$, are deposited on $\mathrm{Rh}(111)$, provide an unambiguous structural and chemical identification of the $7 C_{6}$ building blocks.

\section{KEYWORDS}

Graphene, rhodium, coronene, carbon, STM, DFT, nucleation, growth
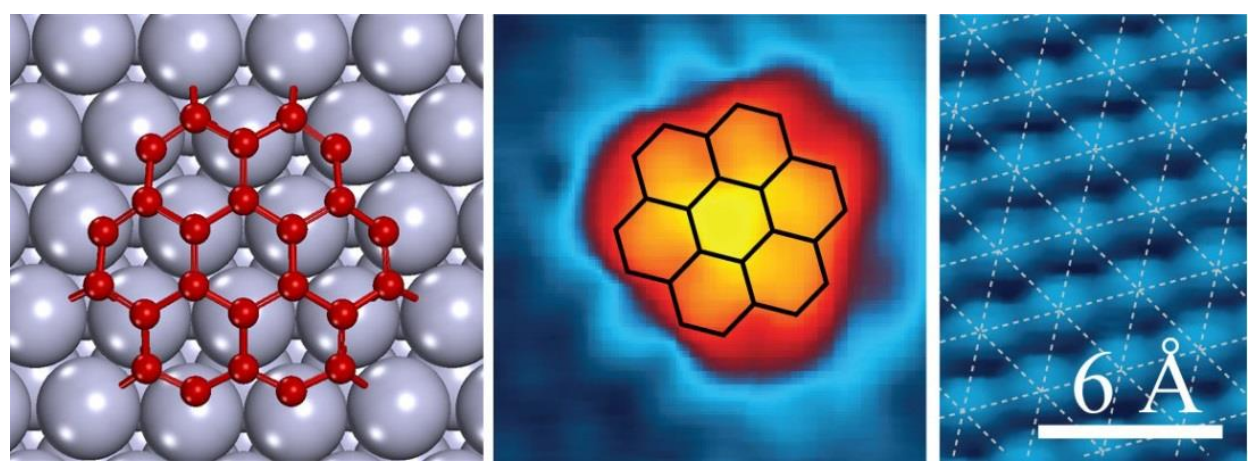

DOI: dx.doi.org/10.1021/nl103053t

Received:

August 29, 2010

Revised:

November 25, 2010

Published:

January 19, 2011 
Ever since its experimental isolation at the turn of the new millennium, graphene has fascinated physicists due to its unique electronic structure. ${ }^{1}$ But more recently, the chemistry community has also realized its potential significance: During a typical heterogeneous catalytic cycle, the reaction of a carbon source (carbon oxides or hydrocarbons) at the surface of an active transition metal (TM) can lead to the formation of various condensed carbonaceous phases. Carbidic or graphitic in nature (as for the one-atom-thick graphene), depending on the interaction strength with the metal catalyst, these carbon forms can be either beneficial or detrimental, by playing an active role in the chemical conversion or by leading to the deactivation (poisoning or coking) of the catalytic sites. ${ }^{2}$ Understanding the formation and development of these various phases on the surfaces of relevant materials, and assessing their stability and reactivity, proves hence to be of utmost importance. ${ }^{3}$

The synthesis of graphene can be accomplished by two complementary approaches based on the hightemperature pyrolysis of small hydrocarbons on TM surfaces: temperature programmed growth (TPG) and chemical vapor deposition (CVD), as extensively demonstrated for (we restrict our discussion below to the $4 d$ and $5 d$ TMs): $\operatorname{Ru}(0001){ }^{4-6} \operatorname{Rh}(111){ }^{7,8}$ $\operatorname{Pd}(111),{ }^{9} \operatorname{Ir}(111),{ }^{10-12}$ and $\mathrm{Pt}(111) .{ }^{13,14}$ Both synthesis methods lead to single-layer graphene characterized by a Moiré superstructure, irrespective of the carbon source (ethene, propene, or benzene) or of the TM support. $^{12,15,16}$ By means of the TPG method, in which the sample is first exposed to the carbon source (e.g., at $300 \mathrm{~K}$ ) and then subsequently annealed to the desired temperature (above $900 \mathrm{~K}$ ), an incomplete overlayer composed of graphene islands is obtained, the maximum coverage of which is defined by the initial carbon uptake at saturation. The size of the carbon islands, ranging from a few to hundreds of nanometers, can be tuned by controlling the growth temperature, and Smoluchowski ripening of small, yet unidentified, carbon nanoislands was reported to govern graphene growth at about $900 \mathrm{~K}^{12} \mathrm{~A}$ recent photoelectron spectroscopy study showed that a strong C-TM interaction exists for the intermediate carbidic species on $\operatorname{Ir}(111)$ by TPG but disappears when these condense into graphitic islands at higher temperature. ${ }^{17}$ The authors explained with the help of DFT calculations that the carbidic clusters bind strongly to the metal substrate through their peripheral atoms, forcing the clusters to adopt a domelike shape. When the carbon source is dosed onto a sample kept at a high temperature, as in the CVD method, the synthesis of an extended, singlelayer graphene covering up to $100 \%$ of the substrate is achieved. No direct evidence of carbon nanoislands has been reported for this approach. Nevertheless, the observation of nonlinear growth kinetics on $\mathrm{Ru}(0001)$, extracted from low energy electron microscopy (LEEM) data, ${ }^{18}$ strongly suggests that the formation of graphene proceeds by incorporation of clusters of approximately $5 \mathrm{C}$ atoms rather than monomers. The attachment energy of an isolated $\mathrm{C}$ atom to an edge of graphene is prohibitively high, whereas it decreases significantly for larger C clusters. ${ }^{18}$

A consensus emerges from these previous studies: Graphene growth involves a series of complicated reactions, whereby dehydrogenation of the carbon source occurs at relatively low temperatures $(<800$ $\mathrm{K})$, followed and/or paralleled by a transition from carbidic species into a graphitic film at higher temperatures (>900 K). Yet, many aspects of the growth mechanism(s) are not fully understood. Although observed by several research groups, the fundamental carbidic building blocks have not been formally identified and little is known about their atomic-scale structure, thermal stability, and electronic properties. The atomistic processes conducive to the formation of a weakly bonded graphitic overlayer based on the merger of strongly interacting carbon species still remain elusive and incomplete. Addressing these issues constitutes a pivotal requirement for our ability to create graphene in a well-controlled and reproducible manner, to tailor the physical and chemical properties of graphene-based nanoscale devices (as of interest to physicists), and to devise strategies either promoting the stability or suppressing the formation of the various carbon phases on TM-based catalysts (as of interest to chemists).

In this report, the decomposition process of carboncontaining molecules (ethene) on $\mathrm{Rh}(111)$ following the TPG method (the CVD method is inaccessible to our experimental facility) was investigated by lowtemperature scanning tunneling microscopy (STM) and density functional theory (DFT) calculations. Our microscopy images reveal, in agreement with studies on other TM substrates ( $\mathrm{Ru}, \mathrm{Ir}, \mathrm{Pt}$ ), that TPG leads to the synthesis of graphene nanoislands with a size of several hundreds of nanometers. We experimentally identify, prior and during the initial stages of graphene island nucleation, carbon nanoclusters of a perfectly monodispersed size. The coarsening of these clusters results in the growth of graphene (a mechanism known as Smoluchowski ripening). STM and STS imaging establish that the carbon nanoislands adopt a domelike shape with an exclusive honeycomb structure composed of exactly seven fused benzene units, hereafter labeled $7 C_{6}$. 
These observations are fully supported by DFT calculations, which further highlight that the enhanced stability of $7 C_{6}$ arises from a subtle clustersize-dependent balance between $\mathrm{C}-\mathrm{C}$ and $\mathrm{C}$-metal bonding. Control experiments and calculations, whereby the hydrogenated analogues of $7 C_{6}$ (i.e., coronene molecules) are deposited onto $\mathrm{Rh}(111)$, not only provide strong support to the identification of $7 C_{6}$ but allow us to further our understanding of the chemical bonding of carbon clusters with a TM substrate. We finally discuss the formation of $7 C_{6}$ as a result of the agglomeration of $C_{2}$ hydrocarbon units.

All experiments were performed in a surface analysis system (in an ultrahigh vacuum environment, UHV, with a base pressure below $1 \times 10^{-10} \mathrm{mbar}$ ) consisting of a preparation chamber allowing for standard sample preparation and characterization by AES, and a microscope chamber housing a CreaTec lowtemperature STM. The Rh(111) crystal was cleaned by repeated cycles of $\mathrm{Ar}^{+}$sputtering and annealing in oxygen $\left(3 \times 10^{-7} \mathrm{mbar}\right)$ at $1100 \mathrm{~K}$ and finalized by flash-annealing in vacuum at $1200 \mathrm{~K}$. STM and STS were performed at liquid helium temperature using the constant-current mode and homemade $\mathrm{W}$ and Ptlr tips. The cleanliness of the surface was monitored by STM and AES. Graphene was prepared by exposure of the $\mathrm{Rh}$ surface to $\mathrm{C}_{2} \mathrm{H}_{4} \quad(99.995 \%$ purity) at room temperature (RT) followed by sequential annealing up to $973 \mathrm{~K}$ in UHV. Coronene molecules were deposited at $\mathrm{RT}$ on $\mathrm{Rh}(111)$ by a homemade evaporator.

During the acquisition of differential conductance maps of the surface (or dl/dV images), standard lockin detection techniques were utilized, ${ }^{19}$ whereby the feedback loop was kept active in order to maintain the tunneling current at a constant value. Topographic and $\mathrm{dl} / \mathrm{dV}$ signals were simultaneously acquired at each pixel (the frequency of the voltage modulation is set to be higher than the bandwidth of the feedback system, hence the tip-sample distance does not react to the modulation when the $\mathrm{dl} / \mathrm{dV}$ signal is acquired at each pixel of the topographic image). To ensure that STM tips were clean and suitable for measurements on carbon nanostructures, STS spectra were first recorded on bare $\mathrm{Rh}(111)$. Only those tips capable of yielding electronically featureless and smooth signatures corresponding to the clean metal surface were utilized.

The DFT calculations were performed with the Vienna Ab-initio Simulation Package (VASP) code, ${ }^{20,21}$ using projector augmented wave (PAW) potentials ${ }^{22}$ and the generalized gradient approximation (GGA-
PBE) for the exchange-correlation functional. ${ }^{23}$ An energy cutoff of $400 \mathrm{eV}$ was used to expand the wave functions into the plane-wave basis. The surfaces were modeled by employing the repeated slab geometry composed of five metal layers in the slab and a vacuum region of about $15 \AA$, on which single $C$ atoms (or $C$ clusters) were adsorbed on one side of the slab. The adsorbate and the topmost two layers were relaxed during structure optimization until the residual forces on the atoms were less than $0.02 \mathrm{eV} / \AA$ ( or $0.05 \mathrm{eV} / \AA ̊ \AA)$. For $\mathrm{C}$ clusters, we used $(4 \times 4),(6 \times 6)$, or $(7 \times 7)$ unit cells according to the cluster size, and used $(3 \times 3 \times 1),(2 \times 2 \times 1),(1 \times 1 \times 1)$ gamma centered $\mathrm{K}$ points, respectively, for the surface Brillouin zone sampling. All parameters defining the numerical accuracy of the calculations were carefully tested. STM images were simulated by using the Tersoff-Hamann approximation, ${ }^{24}$ in which the tunneling current is considered to be proportional to the integrated local density of states (LDOS) within a given energy window determined by the applied bias on the sample. The positive (negative) bias indicates that empty (occupied) states are imaged, in line with what is adopted in our STM measurements.

Figure 1a displays STM images of a $\mathrm{Rh}(111)$ surface saturated with 18 Langmuirs of $\mathrm{C}_{2} \mathrm{H}_{4}$ at $\mathrm{RT}$ and annealed to $473 \mathrm{~K}$. It can be seen that molecular adsorption has induced the well-known formation of the two coexisting $(2 \times 2)$ and $c(4 \times 2)$ superstructures. ${ }^{25}$ Upon deposition at $\mathrm{RT}$, ethene readily deprotonates to ethylidyne $\left(\mathrm{C}_{2} \mathrm{H}_{3}\right)$ and adsorbs in the 3-fold hexagonal close packed (hcp) hollow site with its $\mathrm{C}-\mathrm{C}$ axis perpendicular to the surface. ${ }^{26}$ Annealing the system to $773 \mathrm{~K}$ in UHV (Figure 1b) results in the appearance of small protrusions located on the substrate terraces with sizes ranging from 1 to $2 \mathrm{~nm}$ and an apparent height of less than $0.2 \mathrm{~nm}$. Further successive annealings up to $973 \mathrm{~K}$ (Figure $1 \mathrm{c}, \mathrm{d}$ ) lead to a remarkable narrowing of the particle size distribution and a decrease in the particle density. As can be seen from the high magnification STM image presented in the inset of Figure 1c, the size distribution collapses into a single cluster size of $1 \mathrm{~nm}$ in diameter. This decay coincides with the emergence of graphene islands not only attached to the step edges but also occasionally found on the terraces of the substrate (especially on large terraces). The graphene islands are easily recognizable from their typical Moiré patterns. $6,10-12,14$ Figure 1e shows the density variation of the $1 \mathrm{~nm}$ sized islands (which we will later identify as $7 C_{6}$ ) with annealing temperature. 

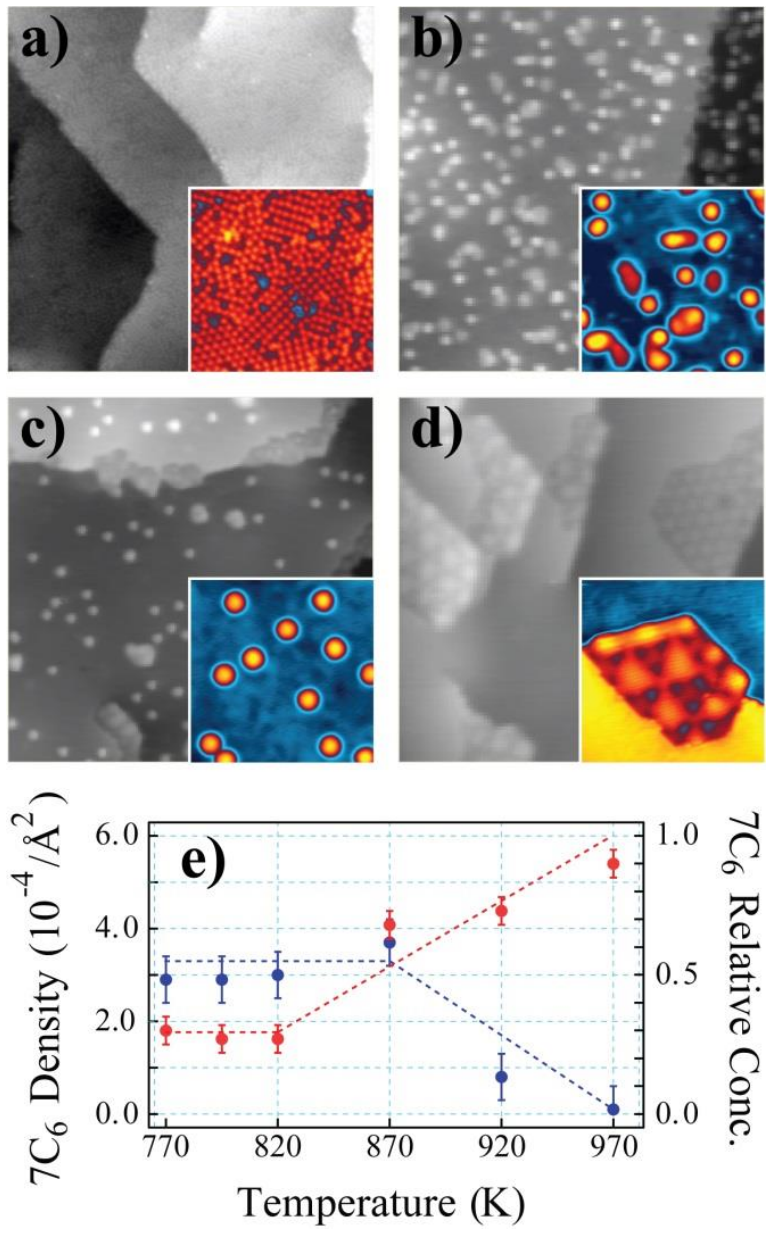

Figure 1: (a-d) STM topographs of the $\mathrm{Rh}(111)$ surface saturated with ethene at room temperature followed by anneals to increasing temperatures $(470,770,870,970 \mathrm{~K}$, respectively). All STM images $400 \times 400 \AA^{2}$ with insets $120 \times 120 \AA^{2}$. (e) Temperature evolution of the surface density of $7 C_{6}$ (in blue) and the relative concentration of $7 \mathrm{C}_{6}$ among all carbon particles identified (in red). The dashed lines are guides-to-the-eye.

The results presented in Figure 1 indicate that thermal decomposition of ethene at temperatures below $770 \mathrm{~K}$ leads to the formation of surface carbon species. ${ }^{25}$ This involves structural rearrangements of $\mathrm{C}-\mathrm{C}$ bonds and dehydrogenation ( $\mathrm{C}-\mathrm{H}$ bond scission). This evolution is fully in line with similar measurements of ethene decomposition on Pt(111). ${ }^{27}$ But remarkably, the carbon species evolve toward a monodispersed structural entity that is randomly scattered across the entire surface, with no preferential attachment to defects such as step edges. The pronounced decrease in particle density observed at $870 \mathrm{~K}$ further suggests that the monodispersed particles start to diffuse within the $\mathrm{Rh}(111)$ terraces and eventually coalesce to form well-ordered graphene structures, which are mostly (but not exclusively) found attached to step edges. At $973 \mathrm{~K}$ (Figure 1d), the much stronger diffusion (hence higher mobility) of the carbon clusters is accompanied by further growth of graphene islands to almost completion. As directly observed in Figure 1c (and confirmed by Figure 1e), one realizes that at high temperatures $7 C_{6}$ is the only species present on the $\mathrm{Rh}(111)$ surface besides graphene flakes. This clearly indicates that $7 C_{6}$ is the unique intermediate precursor involved in the growth of graphene.

The morphology evolution observed in panels b-d in Figure 1 can hence be described by Smoluchowski ripening (that is, the decline of island density through diffusion and coalescence of mobile islands upon contact), in line with previous conclusions drawn for other TM substrates such as Ru and Ir. ${ }^{12,15,16}$ More specifically, the high diffusivity of the $1 \mathrm{~nm}$ carbon clusters accounts for their reduction in population observed above $870 \mathrm{~K}$. During their surface migration, the nanoislands encounter step edges where their attachment is enhanced and their mobility likely decreased, allowing for extended graphene islands to grow by coalescence and incorporation of further carbon nanoislands. A minority of isolated graphene islands are also observed only on very large terraces, indicating that the rate-determining step in graphene formation is coalescence of carbon clusters and not stabilization by step edges or other defects on $\mathrm{Rh}(111)$. The increase of graphene island separation along step edges with temperature is further evidence that nucleation is homogeneous and not restricted to or entirely governed by specific defect sites.
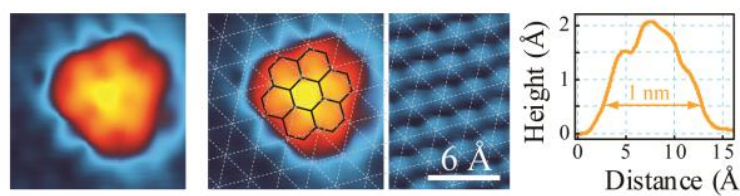

Figure 2: STM topography of $7 \mathrm{C}_{6}\left(15 \times 15 \AA^{2},+0.1 \mathrm{~V}, 0.8 \mathrm{nA}\right)$ accompanied with an atomically resolved STM image of $\mathrm{Rh}(111)$ for visual impression of the atomic scale. A height profile acquired on $7 \mathrm{C}_{6}$ along a high symmetry direction of $\mathrm{Rh}(111)$.

We can estimate the number of carbon atoms deposited on the $\mathrm{Rh}(111)$ surface upon saturation with ethene using STM images similar to Figure 1 . Our statistical analysis yields $6.5 \times 10^{14}$ carbon atoms $/ \mathrm{cm}^{2}$ (with a $\mathrm{Rh}(111)$ lattice parameter of 2.69 $\AA$ ). Furthermore, images such as Figure 1d allow us to estimate that the area of the $\mathrm{Rh}(111)$ surface covered by graphene at completion of the TPG is $18 \pm 2 \%$. Considering a C-C bond distance of $1.42 \AA$ for graphene (i.e., $3.83 \times 10^{15}$ carbon atoms $/ \mathrm{cm}^{2}$ ), we deduce that $(6.9 \pm 0.7) \times 10^{14}$ carbon atoms $/ \mathrm{cm}^{2}$ are involved in our graphene islands. The good agreement between the number of carbons 
deposited and the number of carbons involved in graphene formation confidently rules out the possibility of carbon dissolution into the bulk of the Rh crystal - a conclusion supported by the DFT calculations shown later. We also expect bulk dissolution to be negligible at the temperatures considered here on the basis of similar conclusions reached for the growth of graphene on $\operatorname{Ir}(111),{ }^{12}$ a surface comparable to $\mathrm{Rh}(111)$. Furthermore, the loss of carbon to the gas phase is also negligible during our annealing procedures. ${ }^{25}$
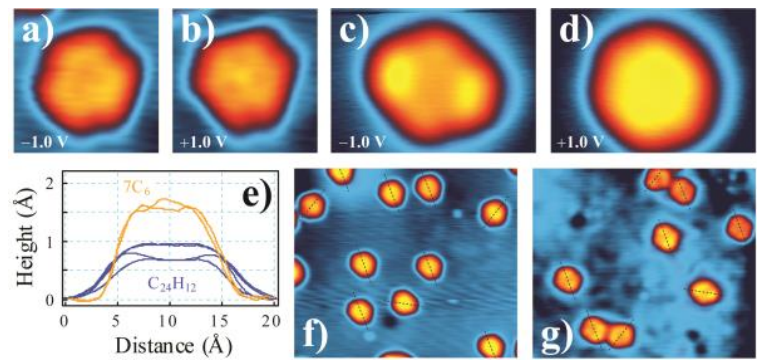

Figure 3: a)-b) STM topographs of a $7 C_{6}$ acquired at -1.0 and $+1.0 \mathrm{~V}$ respectively. $\left.\left(15 \times 15 \AA^{2}, 0.5 \mathrm{nA}\right) . \quad \mathrm{c}\right)$-d) STM topographs of a coronene molecule acquired at -1.0 and $+1.0 \mathrm{~V}$ respectively. $\left(21 \times 15 \AA^{2}, 0.1 \mathrm{nA}\right)$. e) Height profiles acquired on $7 C_{6}$ and coronene along the high symmetry directions of $\mathrm{Rh}(111)$ and extracted from images a) to d). f)g) Large scale STM topographs of $7 \mathrm{C}_{6}$ and coronene respectively $\left(100 \times 85 \AA^{2}\right)$ with their identified molecular alignments with respect to $\mathrm{Rh}(111)$ indicated by dashed lines. Note that images a) to d) have an identical scaling in order to allow for direct topographic comparison, whereby coronene is imaged larger than $7 \mathrm{C}_{6}$.

As mentioned above, a close inspection of the size distribution of the carbon clusters at different temperatures reveals a lower limit, or critical size, at a diameter of $1 \mathrm{~nm}$. From the high magnification image presented in Figure 2, the critical carbon clusters are seen to be nearly perfectly hexagonal in shape. Recognizing that dehydrogenation of ethene is complete at temperatures above $770 \mathrm{~K},{ }^{25}$ the most probable chemical structure adopted by the nanoclusters is comprised of seven honeycomb carbon units (or equivalently seven benzene units fused together and stripped from their protons) arranged in a hexagon, hereafter labeled $7 \mathrm{C}_{6}$. From the height contrast in the STM image of $7 C_{6}$ and from the measured height profile (Figure 2), one gets the visual impression (under these tunneling conditions) that the central benzene unit lies higher than its surrounding neighbors, evocative of a domelike shaped configuration with a total height of about $2 \AA$. By resolving the atomic lattice of the substrate around the carbon cluster (see Figure 2), the preferred adsorption site of $7 C_{6}$ (defined as the substrate site directly below the central benzene ring) turns out to be the face-center cubic (fcc) hollow site. We stress here that we were only able to acquire a handful of images (about 10) in which atomic resolution of the substrate was achieved without evidence of any disturbance to the carbon clusters. While in all cases the favored adsorption site was indeed identified as fcc hollow, the statistical confidence in this result is not optimal. These observations will, however, be substantiated later by DFT calculations.

In order to fully establish the structural and chemical identity of these carbon nanoclusters, and to discover the reasons of this lower size limit, we now proceed to further STM data, local spectroscopy measurements, and DFT calculations. Our experimental and theoretical results on $7 \mathrm{C}_{6}$ will be compared to investigations we carried on coronene molecules $\left(\mathrm{C}_{24} \mathrm{H}_{12}\right)$ adsorbed on $\mathrm{Rh}(111)$, the hydrogenated analogues of $7 C_{6}$, allowing also for conclusions to be drawn on the effect of hybridization and hydrogen functionalization on the chemical bonding of nanographene molecules with the TM substrate.

In Figure 3, we put side by side topographic images of $7 C_{6}$ (obtained by the thermal decomposition of ethene at high temperatures) and coronene (obtained by molecular evaporation at $300 \mathrm{~K}$ ) on $\mathrm{Rh}(111)$. Direct comparison indicates that, irrespective of the tunneling bias applied ( -1 or $+1 \mathrm{~V})$, coronene molecules are imaged broader than $7 C_{6}$ whereas the electronic height of the latter is larger by $0.5 \AA$ (see height profiles in Figure 3e). At both positive and negative bias, $7 C_{6}$ exhibits a slightly truncated hexagonal shape with a polaritydependent orientation. The lateral dimensions of the carbon cluster, as discussed for Figure 2, reflect the ones expected for the $7 \mathrm{C}_{6}$ molecular skeleton. It is hence not a surprise to observe slightly larger lateral dimensions for coronene in view of the protons surrounding the carbon skeleton. Most importantly, the topographic contrast obtained over coronene is markedly different to the one obtained over $7 \mathrm{C}_{6}$. At positive polarity, coronene is imaged as hexagonal, whereas at negative polarity, it is imaged with a richer contrast whereby the molecule is resolved as a slightly distorted hexagon but, surprisingly, with two intense and broad lobes on two opposite sides. The presence of the latter renders the coronene molecules 2 -fold symmetric when observed by STM at negative bias, whereas $7 C_{6}$ exhibits a 3 -fold symmetry. Indeed, this is the case as illustrated in panels $f$ and $g$ of Figure 3. These symmetry arguments strongly suggest that coronene preferentially adsorbs on a $\mathrm{Rh}(111)$ bridge site, whereas, as we concluded earlier, the 3 -fold symmetry of $7 C_{6}$ arises from adsorption at the fcc 
hollow site. In essence, the results presented in Figure 3 provide a first experimental indication that the carbon clusters evolving from ethene decomposition on $\mathrm{Rh}(111)$ are not comparable to coronene molecules and their likely molecular structure is hence the one we ascribed to $7 C_{6}$, that is, a fully dehydrogenated and 3-fold symmetric carbon nanoisland of precisely 24 carbon atoms.
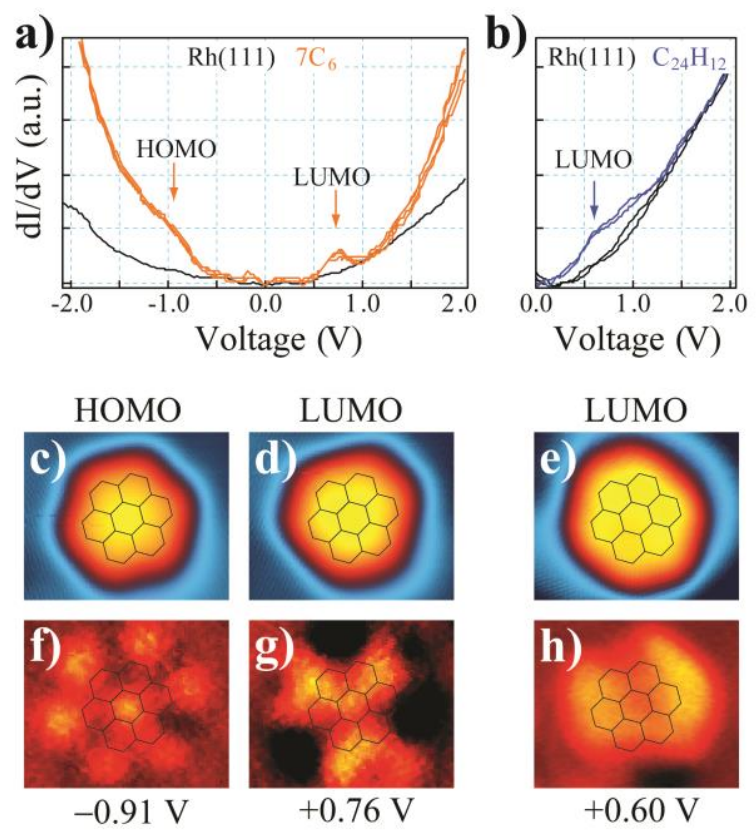

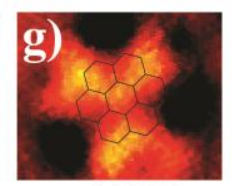

$+0.76 \mathrm{~V}$
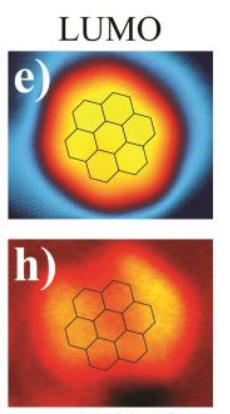

$+0.60 \mathrm{~V}$
Figure 4: (a) STS spectra acquired on $7 \mathrm{C}_{6}$. (b) STS spectra acquired on coronene. (c, d) Topographic STM images acquired on $7 C_{6}$ at the voltages corresponding to the HOMO and LUMO peaks identified in (a). (e) Topographic image of coronene acquired at the voltage associated with the LUMO peak identified in (b). ( $f, g$ ) Differential conductance maps acquired on $7 C_{6}$ corresponding to (c, d). (h) Differential conductance map acquired on coronene corresponding to (e). All STM images $17 \times 14 \AA^{2}$.

Parts $a$ and $b$ of Figure 4 show typical $d l / d V$ spectra recorded on the centers of $7 C_{6}$ clusters and coronene molecules adsorbed on $\mathrm{Rh}(111)$, respectively. The $7 C_{6}$ spectra are characterized by a broad peak located at $-0.91 \mathrm{~V}$ and a sharp peak at +0.76 $\mathrm{V}$ (easily identifiable when comparing with spectra acquired on the bare Rh substrate) that we associate with the HOMO and LUMO states, respectively. The coronene spectrum exhibits a single broad peak near $+0.6 \mathrm{~V}$ while no obvious peak or shoulder is observed at negative bias. The position and the width of these features vary slightly from one cluster (or molecule) to the other. The striking dissimilarity between the spectra in parts $a$ and $b$ of Figure 4 is a further indication that $7 C_{6}$ and coronene are electronically different entities.

At $-0.91 \mathrm{~V}$, corresponding to the $7 \mathrm{C}_{6} \mathrm{HOMO}$ resonance identified in Figure $4 a$, the cluster is imaged by constant-current STM as a slightly truncated hexagon with a height of approximately $1.5 \AA$ (Figure 4c). No intramolecular structure is resolved. At a positive bias of $+0.76 \mathrm{~V}$ associated with the LUMO energy, $7 C_{6}$ is again imaged as a slightly truncated hexagon although with a rotation of $180^{\circ}$ (Figure 4d). Again, no intramolecular structure is observed. STM images of coronene molecules (Figure 4e), obtained at $+0.6 \mathrm{~V}$, corresponding to the single resonance identified in Figure $4 b$, are similar to those for $7 C_{6}$, although slightly larger and reflecting the overall shape and size of the molecule, with a reduced height of approximately $1 \AA$ as compared to $7 \mathrm{C}_{6}$. No intramolecular details can be discerned. Note that when coronene molecules are imaged at voltages within their featureless, negative spectral region, we obtain similar images as the one discussed and presented in Figure $3 c$. In panels $f, g$, and $h$ of Figure 4 , we present energy-resolved spectroscopic maps of the $7 C_{6}$ cluster and the coronene molecule taken at the energies of their respective resonances identified in spectra $a$ and $b$ of Figure 4 . The $d l / d V$ map of the highest energy resonance of the carbon cluster $(+0.76 \mathrm{~V}$, Figure $4 \mathrm{~g})$ displays three nodal structures with $120^{\circ}$ separation, while the map of the lowest energy state at $-0.91 \mathrm{~V}$ (Figure $4 \mathrm{f}$ ) shows a network of seven LDOS peaks located at the expected sites of seven hexagon rings. In contrast, no intramolecular spatial inhomogeneity in the electronic structure can be distinctively observed for coronene (Figure 4h). Although the spectroscopic maps of $7 C_{6}$ clearly retain the 3 -fold symmetry deduced from the topographic images, the 2-fold symmetric signature of coronene is just recognizable.

To understand the nucleation and growth of graphene from a theoretical point of view, we need to consider three predominant forms of carbon interacting with the $\mathrm{Rh}$ substrate and their respective formation: (1) single, isolated $\mathrm{C}$ adatoms, (2) subsurface $C$ atoms, and (3) aggregates of C atoms such as clusters and graphene. For the adsorption of carbon adatoms, we studied by DFT six different coverages: $1 / 9 \mathrm{ML}, 1 / 6 \mathrm{ML}, 1 / 4 \mathrm{ML}, 1 / 2$ $\mathrm{ML}, 3 / 4 \mathrm{ML}$, and $1 \mathrm{ML}$. For the favorable hcp adsorption site, the corresponding calculated adsorption energies with respect to atomic carbon in the gas phase are -7.37, -7.33, -7.25, -6.62, -6.19, and $-5.64 \mathrm{eV}$, respectively. Decreasing adsorption energies with coverage indicates that the lateral interaction between $C$ adatoms is repulsive. For subsurface carbon, 1/6 ML and 1/4 ML coverages were investigated. The octahedral site is preferred, with corresponding adsorption energies of -7.11 and $-6.91 \mathrm{eV}$, respectively. The comparison of the 
energetics for $C$ adatoms and subsurface $C$ atoms at equivalent coverage indicates that adsorption on the surface is energetically favorable, in line with our experimental observations that no carbon is lost to the bulk during growth. The computed formation energy for free-standing graphene with respect to atomic carbon in the gas phase amounts to $-7.90 \mathrm{eV}$. The fact that this value is lower than that of single $C$ atoms adsorbed on the surface, even without accounting for the interfacial interaction between graphene and the Rh substrate, constitutes the thermodynamical driving force toward graphene formation on $\mathrm{Rh}(111)$. Let alone, however, this deduction does not explain the occurrence of size selective carbon clusters during growth.

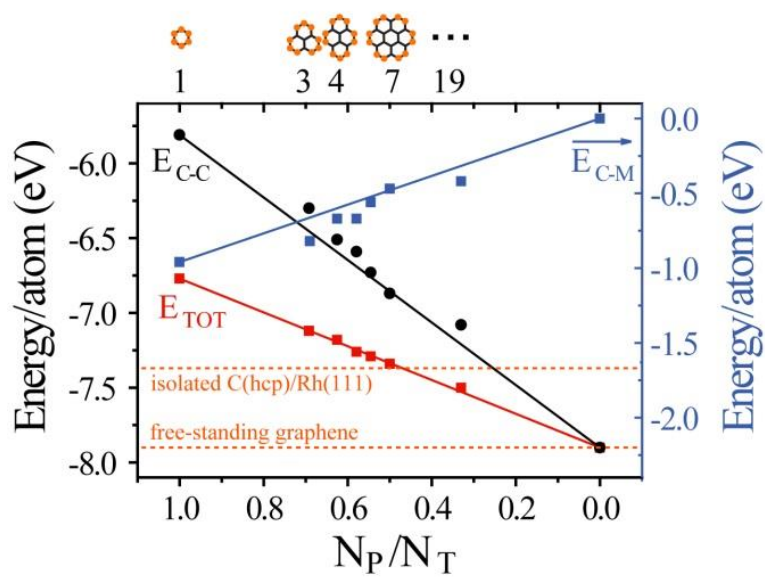

Figure 5: DFT computed formation energies for $\mathrm{NC}_{6}$ clusters adsorbed on $\mathrm{Rh}(111)$ as function of cluster size ( $N$ $=1,3,4,5,6,7$, and 19). $E_{\mathrm{TOT}}$ (in red), $E_{\mathrm{C}-\mathrm{M}}$ (in blue), and $E_{\mathrm{C}-\mathrm{c}}$ (in black). See text for detailed explanations.

In order to shed more light, we theoretically investigated the adsorption of $N C_{6}$ carbon clusters on the Rh(111) surface, where $N$ indexes the number of honeycomb rings contained within each cluster. The results are reported in Figure 5. The top and bottom $\mathrm{x}$ axes display $N$ and the ratio between the number of peripheral carbon atoms $\left(N_{\mathrm{P}}\right)$ and the total number of $C$ atoms in the $N C_{6}$ clusters $\left(N_{\mathrm{T}}\right)$, respectively. The $y$ axis shows the total energy gain ( $E_{\mathrm{TOT}}$, per carbon atom, represented by the red dots in Figure 5) of the carbon species with respect to atomic carbon in the gas phase, which results from two separate contributions: the formation energy of the clusters in gas phase assumed in their adsorbed configuration ( $E_{\mathrm{C}-\mathrm{c}}$ per carbon atom, represented by the black dots) and the adsorption energy of the clusters on $\mathrm{Rh}(111)$ ( $E_{\mathrm{C}-\mathrm{M}}$ per carbon atom, represented by the blue dots). For reference, the two orange dashed lines in Figure 5 indicate the adsorption energy of the isolated atomic carbon at $1 / 9 \mathrm{ML}$ coverage (that is, in its most isolated configuration) and the formation energy of free- standing graphene. Note that the energy values of the adsorbed carbon monomer or clusters reported here correspond to the preferred adsorption geometries (see discussion below).

Figure 5 reveals that the atoms at the periphery of the carbon species constitute the important structural parameter governing the interaction of $N C_{6}$ with the $\mathrm{Rh}(111)$ substrate, in line with a recent theoretical report. ${ }^{17}$ Indeed, we see that both $E_{\mathrm{C}-\mathrm{M}}$ and $E_{\mathrm{C}-\mathrm{C}}$ show a linear dependence (with opposite trend) with the ratio $N_{\mathrm{P}} / N_{\mathrm{T}}$. In particular, $E_{\mathrm{C}-\mathrm{M}}$ decreases with $N_{\mathrm{P}} / N_{\mathrm{T}}$ indicating that the $N C_{6}$ species bind to the $R h(111)$ substrate mainly through the peripheral atoms. We also note that $E_{\mathrm{C}-\mathrm{c}}$ increases with $N_{\mathrm{p}} / N_{\mathrm{T}}$ since less peripheral atoms with dangling bond are present. A maximum value is naturally reached when the formation of extended graphene on $\mathrm{Rh}(111)$ is complete, which is represented energetically by the formation energy of freestanding graphene, neglecting its interaction with the substrate (the latter amounts to only a few millielectronvolts $\left.{ }^{8}\right)$. Interestingly, when comparing the variation of $E_{\text {ТОт }}$ as a function of $N$ with reference to the corresponding energy of the isolated carbon adatom in Figure 5, it becomes apparent that the aggregation of carbon into clusters equal or larger in size than $7 C_{6}$ is energetically favored. In other words, $7 C_{6}$ is the smallest stable carbon cluster on $\mathrm{Rh}(111)$. Since clusters larger than $7 C_{6}$ have even lower energies, we conclude that the $7 C_{6}$ species will eventuallygrow into larger graphene islands at high enough temperatures. These theoretical conclusions remarkably support our STM observations of $7 C_{6}$ clusters obtained by the dehydrogenation of ethene on $\mathrm{Rh}(111)$ and allow us to conclude that these monodispersed species are the fundamental precursors to the formation of graphene on a $\mathrm{Rh}(111)$ substrate. Note that a minimum in $E_{\text {Tот }}$ for $7 C_{6}$ is not to be expected, since thermodynamics drives the C-M system toward the formation of epitaxial graphene (for which $E_{\mathrm{TOT}}$ is minimum). Furthermore, the averaged energy difference between free-standing graphene and graphene/ $\mathrm{Rh}(111)$ is negligible ( $8 \mathrm{meV}$ per $\mathrm{C}$ atom).$^{8}$

We now concentrate specifically on calculations performed for $7 C_{6}$ and coronene to identify bonding differences between the two species. The relaxed geometries for $7 \mathrm{C}_{6}$ on different adsorption sites (hollow, top and bridge) on $\mathrm{Rh}(111)$ are reported in Figure 6a. Our experimental observation of 3-fold symmetric $7 C_{6}$ clusters suggested adsorption at either top or hollow sites (high-resolution STM images identified fcc sites, Figure 2), safely excluding bridge sites. Calculated formation energies for $7 C_{6}$ at the top site are $0.02 \mathrm{eV}$ per carbon atom lower than 
that of the hollow sites, indicating that $7 \mathrm{C}_{6}$ adsorbed at the top site is preferred. It is worth noting that van der Waals interactions are not properly described in the present exchange-correlation functional ${ }^{28}$ and may affect the overall energetics and relative differences of $7 C_{6}$ at different sites. Nevertheless, a striking structural feature seen from Figure 6a is immediately apparent: irrespective of the adsorption sites, the carbon clusters adopt a dome-like shape, due to the exclusive bonding of the peripheral carbon atoms to the $\mathrm{Rh}(111)$ substrate, and beautifully complementing the interpretation of the STM contrast observed in Figure 2. For the top site, the width of adsorbed $7 C_{6}$ amounts to $7.05 \AA$, with a height (measured as the distance between carbon and the Rh surface atoms) between 2.93 (cluster center) and $1.75 \AA$ (cluster edge). For both fcc and hcp sites, the width amounts to $7.43 \AA$, with a height between 2.65 and $1.80 \AA$.

a) $7 \mathrm{C}_{6}$

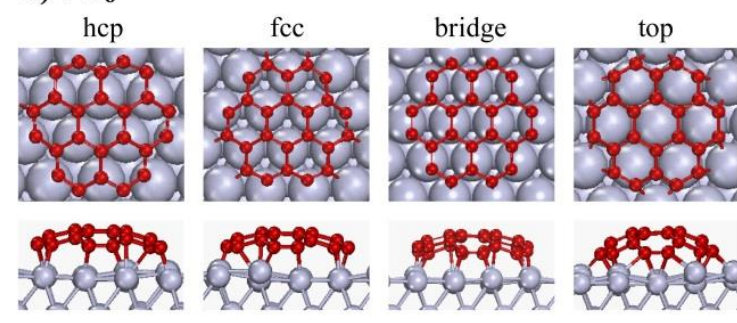

b) coronene

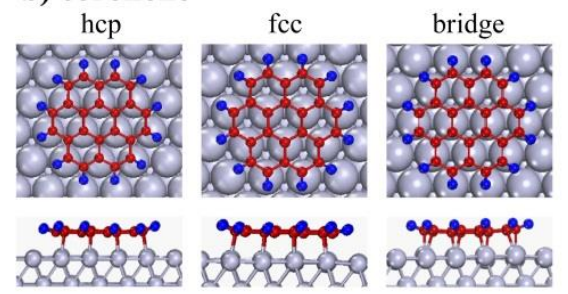

Figure 6: DFT relaxed structures for the adsorption of (a) $7 \mathrm{C}_{6}$ and (b) coronene at various sites on the $\mathrm{Rh}(111)$ surface.

The optimized structures for coronene/Rh(111) are markedly different, as shown in Figure $6 \mathrm{~b}$. We find that the adsorption at the bridge site is more energetically favorable than that at the hollow sites. Irrespective of the adsorption sites, the width of the carbon skeleton of coronene is $7.5 \AA$, and the carbon atoms lie parallel to the surface with heights of 0.50 (bridge) and $0.41 \AA$ ( $\mathrm{hcp}$ and fcc) lower than the center of fcc $7 C_{6}$ on $\mathrm{Rh}(111)$. These results confirm our experimental findings remarkably, even considering the fact that van der Waals interactions are not properly described in the present calculations, which may affect the relative stability. Indeed, when including the contribution of $\mathrm{H}$ atoms to the lateral dimensions, the larger appearance of coronene molecules in our STM topographs is rationalized. Furthermore, the preferred adsorption site (bridge) confirms the 2-fold symmetric appearance of coronene in STM images, and we can expect, based on the C-Rh distances, to image $7 \mathrm{C}_{6}$ higher than coronene.

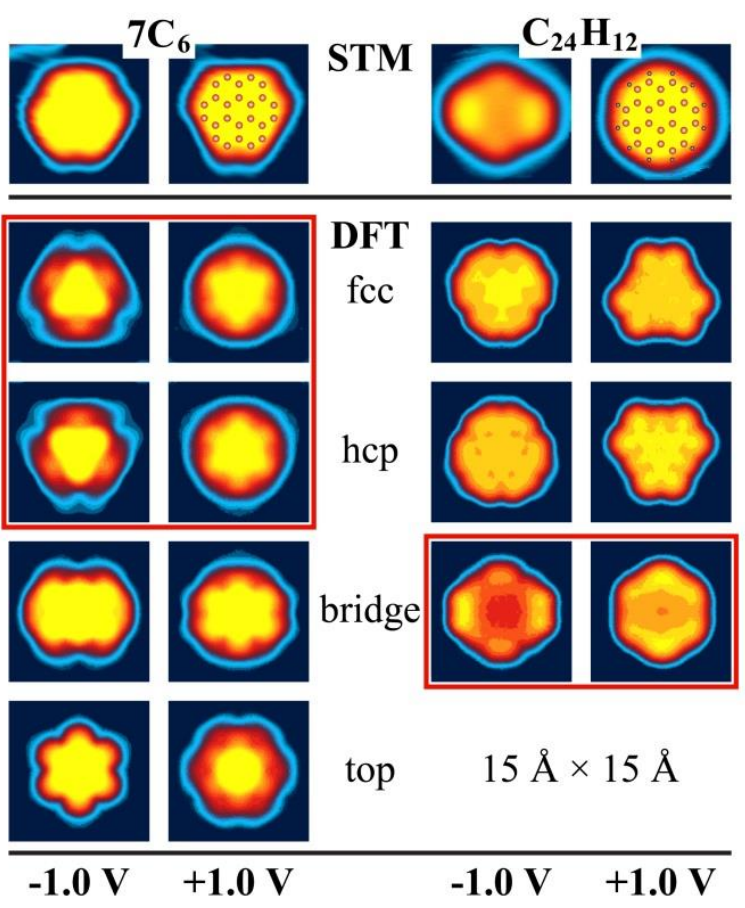

Figure 7: DFT simulated STM images based on the TersoffHamann theory of $7 \mathrm{C}_{6}$ and coronene adsorbed at different sites on the $\mathrm{Rh}(111)$ surface. The first row displays the experimental STM images to allow for a direct comparison with the theoretical images. Left columns correspond to $7 \mathrm{C}_{6}$ and right columns to coronene. The red boxes highlight the computed adsorption configurations best matching the experimental observations. All images are $15 \times 15 \AA^{2}$.

To demonstrate the agreement further, we present in Figure 7 the simulated STM images for $7 C_{6}$ and coronene adsorbed on $\mathrm{Rh}(111)$ at the various adsorption sites. For $7 C_{6}$, the STM simulated images for the hcp and fcc adsorption sites are almost identical: 3-fold symmetric at both negative and positive biases (note the polarity-dependent orientation of the slight truncation), while for the bridge site we recognize the expected (but experimentally unobserved) 2-fold symmetry. The STM simulation for the top site reveals a yet unseen 6-fold symmetry, confidently ruling out this configuration and consequently restricting $7 \mathrm{C}_{6}$ adsorption to a hollow site (fcc or hcp). The theoretical STM topographs for coronene/Rh(111) show a compelling similarity with our experimental images only for adsorption at the bridge site, for which the observed structural features (two bright lobes on adjacent sides) are clearly discernible. 
Furthermore, the size of coronene is imaged broader than that of $7 C_{6}$.

An issue of importance remains yet unanswered: How do the $7 C_{6}$ clusters form on Rh(111)? Our STM data indicate that adsorbed ethylidyne molecules (Figure 1a) evolve between 470 and $770 \mathrm{~K}$ toward hydrocarbon clusters with sizes of about 1-2 nm (Figure $1 \mathrm{~b}$ ). We do not observe smaller protrusions reminiscent of potential $C_{1}$ decomposition fragments (or $\mathrm{CH}_{\mathrm{x}}$ ), which would necessarily involve $\mathrm{C}-\mathrm{C}$ bond breaking. In a recent theoretical paper, Chen et al. ${ }^{29}$ discuss the kinetics and energetics of carbon nucleation in the early stages of graphene epitaxial growth on metal surfaces. For group VIIIb TMs, step sites are required to facilitate the formation of dimers. It is assumed here that the carbon source consists of single $\mathrm{C}$ atoms. If such a mechanism were to operate under our experimental conditions, we would expect to observe carbon clusters exclusively attached to steps, whereas the hydrocarbon complexes and their progeny, $7 C_{6}$, are exclusively found on terrace sites (Figure 1b,c). Although we cannot experimentally rule the existence of these $C_{1}$ fragments during our annealing procedures, it is more likely that ethylidyne polymerizes to the observed heavier hydrocarbon complexes, which in turn decompose to $7 C_{6}$ by hydrogen evolution. We hence speculate that, in a first step, the mobile $C_{2}$ hydrocarbons couple to gradually form larger hydrocarbon species. This growth mechanism is further strengthened by recent XPS measurements ${ }^{30}$ whereby $C^{2}$ units were indirectly postulated to account for graphene growth from acetaldehyde $\left(\mathrm{CH}_{3} \mathrm{CHO}\right)$ and acetone $\left(\mathrm{CH}_{3} \mathrm{CCH}_{3} \mathrm{O}\right)$ on a $\mathrm{Rh}(111)$ thin film. The polymeric structures reach sizes of up to 1$2 \mathrm{~nm}$ while simultaneously evolving $\mathrm{H}$ to the gasphase upon annealing to ever higher temperatures (note that this size is comparable to $7 \mathrm{C}_{6}$ or slightly larger due to the presence of the remaining $\mathrm{H}$ atoms, see discussion above). When full dehydrogenation is attained, the $\mathrm{Rh}(111)$ surface accommodates predominantly the resulting monodispersed $7 \mathrm{C}_{6}$ species, the stability of which is understood on the basis of the theoretical considerations presented above. And in a final, high-temperature step, the later precursors coalesce to graphene flakes, as ultimately predicted by thermodynamics.

In essence, our experimental and theoretical investigations demonstrate that the growth of graphene on $\mathrm{Rh}(111)$, following TPG of ethene, proceeds by coalescence (Smoluchowski ripening) of monodispersed carbon $7 C_{6}$ clusters. The peculiar stability of these species is understood from DFT calculations to arise from a delicate, cluster-sizedependent balance between C-C and C-TM bonding.
The comparison with experiments focusing on the adsorption of coronene molecules shows that, whereas coronene adopts a flat lying structure whereby the bonding to the substrate is delocalized over the molecular $\pi$ backbone, the $7 C_{6}$ precursors are dome-like shaped and essentially bind to the substrate via the peripheral carbon atoms. Our final concern relates to the transferability of our results to the alternative CVD growth method. The fact that Loginova and co-workers observed nonlinear growth kinetics for graphene on $\operatorname{Ir}(111),{ }^{18}$ as we discussed previously, suggests that similar growth mechanisms (i.e., growth by incorporation of carbon clusters rather than monomers) operate under both CVD and TPG conditions. The large difference between the C$\mathrm{C}$ and $\mathrm{C}-\mathrm{H}$ bond strengths naturally favors dehydrogenation and graphitization at elevated temperatures on the VIIIb group TMs. Since the CTM interaction strength varies across these metals and their surface orientation, as predicted by the $d$ band model, ${ }^{31}$ we can however realistically expect differences in size and shape of the precursors depending on the choice of substrate and/or growth methodology.

\section{ACKNOWLEDGEMENTS}

R.S. acknowledges the financial support from the Scottish Funding Council, through EaStCHEM and the SRDG Grant HR07003. W.X.L. is grateful for the financial support from the Natural Science Foundation of China (20873142, 20733008, 20923001) and the Ministry of Science and Technology of China (2007CB815205). We acknowledge fruitful discussions with Professors Xinhe Bao and Qiang Fu.

\section{REFERENCES}

(1) Novoselov, K. S.; Geim, A. K.; Morozov, S. V.; Jiang, D.; Katsnelson, M. I.; Grigorieva, I. V.; Dubonos, S. V.; Firsov, A. A. Nature 2005, 438, 197.

(2) Forzatti, P.; Lietti, L. Catal. Today 1999, 52, 165.

(3) Ruoff, R. Nat. Nanotechnol. 2008, 3, 10.

(4) Marchini, S.; Günther, S.; Wintterlin, J. Phys. Rev. B 2007, 76, No. 075429.

(5) Sutter, P. W.; Flege, J. I.; Sutter, E. A. Nat. Mater. 2008, $7,406$.

(6) Vazquez de Parga, A. L.; Calleja, F.; Borca, B.; Passeggi, M. C. G.; Hinarejos, J. J.; Guinea, F.; Miranda, R. Phys. Rev. Lett. 2008, 100, No. 056807.

(7) Sicot, M.; Bouvron, S.; Zander, O.; Rüdiger, U.; Dedkov, Y. S.; Fonin, M. Appl. Phys. Lett. 2010, 96, No. 093115.

(8) Wang, B.; Caffio, M.; Bromley, C.; Früchtl, H.; Schaub, R. ACS Nano 2010, 4, 5773.

(9) Kwon, S.-Y.; Ciobanu, C. V.; Petrova, V.; Shenoy, V. B.; Bareno, J.; Gambin, V.; Petrov, I.; Kodambaka, S. Nano Lett. 2009, 9, 3985.

(10) N'Diaye, A. T.; Bleikamp, S.; Feibelman, P. J.; Michely, T. Phys. Rev. Lett. 2006, 97, No. 215501. 
(11) Coraux, J.; N'Diaye, A. T.; Busse, C.; Michely, T. Nano Lett. 2008, 8, 565.

(12) Coraux, J.; N'Diaye, A. T.; Engler, M.; Busse, C.; Wall, D.; Buckanie, N.; Meyer zu Heringdorf, F.-J.; van Gastel, R.; Poelsema, B.; Michely, T. New J. Phys. 2009, 11, No. 023006.

(13) Fujita, T.; Kobayashi, W.; Oshima, C. Surf. Interface Anal. 2005, 37, 120.

(14) Land, T. A.; Michely, T.; Behm, R. J.; Hemminger, J. C.; Comsa, G. Surf. Sci. 1992, 264, 261.

(15) Donner, K.; Jakob, P. J. Chem. Phys. 2009, 131, 164701.

(16) Zhang, H.; Fu, Q.; Cui, Y.; Tan, D.; Bao, X. J. Phys. Chem. C 2009, 113, 8296

(17) Lacovig, P.; Pozzo, M.; Alfe, D.; Vilmercati, P.; Baraldi, A.; Lizzit, S. Phys. Rev. Lett. 2009, 103, No. 166101.

(18) Loginova, E.; Bartelt, N. C.; Feibelman, P. J.; McCarty, K. F. New J. Phys. 2009, 11, No. 063046.

(19) Becker, R. S.; Golovchenko, J. A.; Hamann, D. R.; Swartzentruber, B. S. Phys. Rev. Lett. 1985, 55, 2032.

(20) Kresse, G.; Furthmüller, J. Phys. Rev. B 1996, 54, 11169.

(21) Kresse, G.; Furthmüller, J. Comput. Mater. Sci. 1996, 6, 15.

(22) Blöchl, P. E. Phys. Rev. B 1994, 50, 17953.

(23) Perdew, J. P.; Burke, K.; Ernzerhof, M. Phys. Rev. Lett. 1996, 77, 3865.

(24) Tersoff, J.; Hamann, D. R. Phys. Rev. Lett. 1983, 50, 1998.

(25) Dubois, L. H.; Castner, D. G.; Somorjai, G. A. J. Chem. Phys. 1980, 72, 5234.

(26) Wander, A.; Van Hove, M. A.; Somorjai, G. A. Phys. Rev. Lett. 1991, 67, 626.

(27) Land, T. A.; Michely, T.; Behm, R. J.; Hemminger, J. C.; Comsa, G. J. Chem. Phys. 1992, 97, 6774.

(28) Dion, M.; Rydberg, H.; Schröder, E.; Langreth, D. C.; Lundqvist, B. I. Phys. Rev. Lett. 2004, 92, No. 246401.

(29) Chen, H.; Zhu, W.; Zhang, Z. Phys. Rev. Lett. 2010, 104, No. 186101.

(30) Müller, F.; Sachdev, H.;Hüfner, S.; Pollard, A. J.; Perkins, E. W.; Russell, J. C.; Beton, P. H.; Gsell, S.; Fischer, M.; Schreck, M.; Stritzker, B. Small 2009, 5, 2291.

(31) Hammer, B.; Nørskov, J. K. Surf. Sci. 1995, 343, 211. 